both increased odds of negative outcome. Buccal midazolam was used in the management of $28.9 \%$ of seizures and had no effect on the need for ventilatory support. The majority of seizures (69.8\%) required admission to hospital and only $4.0 \%$ resulted in adverse outcome. Of 1228, there were 2 deaths $(0.2 \%)$. Compared to symptomatic seizures, unprovoked seizures had a longer average duration, higher likelihood of negative outcomes and there was a higher proportion of those with other neurological diagnosis.

Conclusions Adverse outcomes have decreased and the use of buccal midazolam is promising. Identifying high-risk groups provides opportunity for early intervention. This data forms the basis for extensive evaluation of acute seizure management and monitoring long-term outcomes.

\section{G43(P) IMPROVING ANTIMICROBIAL TREATMENT OF CHILDREN WITH SUSPECTED ENCEPHALITIS IN A TERTIARY PAEDIATRIC HOSPITAL}

${ }^{1}$ I Husain, ${ }^{2}$ D Lumsden. ' GKT School of Medical Education, King's College London, London, UK; ${ }^{2}$ Paediatric Neurology, Evelina Children's Hospital, London, UK

\subsection{6/archdischild-2020-rcpch.30}

Background/Aims Encephalitis is defined as 'inflammation of the brain parenchyma, manifest by neurologic dysfunction'. It is estimated that there are 2.8 cases per 100,000 children in the UK. Encephalitis can have devastating consequences and children who survive the illness can continue to experience physical, cognitive, mental and social difficulties. However, early appropriate treatment can dramatically improve outcomes. Trust antimicrobial guidelines advise giving $80 \mathrm{mg} / \mathrm{kg}$ intravenous ceftriaxone once daily, intravenous aciclovir (advised doses differ by age) and $7.5 \mathrm{mg} / \mathrm{kg}$ oral clarithromycin twice daily as empirical treatment in children aged over 1 month with suspected encephalitis.

The aim of this audit was to ascertain current management of children with suspected encephalitis in a tertiary paediatric hospital compared to local guidelines.

Methods Our sample included children aged between 28 days and 18 years old with a suspected diagnosis of encephalitis, who had received treatment at our centre over a six-month period. Children were identified from review of Pharmacy records of inpatient prescriptions for acyclovir and/or ceftriaxone. A standardised pro-forma was used to collect data on patient characteristics, investigations and management.

Results Twenty-five children were admitted to either the Paediatric Intensive Care Unit (PICU) or paediatric wards with suspected encephalitis within the six-month period. Two patients had to be excluded from the sample. Eight of the remaining twenty-three children had encephalitis treatment started at other district general hospitals prior to transfer. Only 6/23 (26.1\%) patients fully received the recommended treatment

\begin{tabular}{llll}
$\begin{array}{l}\text { Abstract G43(P) Table 1 } \\
\text { medication }\end{array}$ & Types of prescribing errors for each \\
\hline & $\begin{array}{l}\text { Total number of prescribing } \\
\text { errors }\end{array}$ & $\begin{array}{l}\text { Not } \\
\text { prescribed }\end{array}$ & $\begin{array}{l}\text { Wrong } \\
\text { dose }\end{array}$ \\
\hline Ceftriaxone & 5 & 2 & 3 \\
Aciclovir & 6 & 2 & 4 \\
Clarithromycin & 14 & 8 & 6 \\
\hline
\end{tabular}

for suspected encephalitis. There were errors in prescribing of ceftriaxone, in $5 / 23(21.7 \%)$ patients, aciclovir in $6 / 23$ (26.1\%) patients and clarithromycin in 14/23 (60.9\%) patients. Table 1 shows an overview of the types of prescribing errors. Conclusion Audit results identified that errors in antimicrobial prescriptions for children with suspected encephalitis were common, most frequently for macrolide therapy. We have instigated a poster to be displayed in ward areas, and a crib sheet for PICU and ward healthcare professionals, and plan to reaudit in the near future.

\section{G44(P) SYDENHAM'S CHOREA: A FORGOTTEN ENTITY IN A MODERN WORLD}

S Kurmani, A Neduvamkunnil, M Prasad. General Paediatrics, Queen's Medical Centre, Nottingham University Hospitals, Nottingham, UK

\subsection{6/archdischild-2020-rcpch.31}

Acute Rheumatic Fever (ARF) is a multi-system inflammatory disease which is a rare but serious complication of a Group A Streptococcus throat infection. Over the past 50 years, its incidence has fallen in developed countries due to improvements in medical care and standard of living, affecting less than 1 in 100,000 people in the UK. ARF is no longer a leading contributor to cardiovascular morbidity amongst children. Therefore, the clinical index of suspicion of ARF may be low amongst healthcare professionals, as its clinical features and symptoms may resemble those of more common conditions.

We present a case of a previously fit and well 13-year-old girl, with a history of behavioural changes, emotional lability and increasing restlessness. Initially, her symptoms were attributed to a behavioural or mental health disorder. Clinical examination demonstrated involuntary writhing movements, stereotypical of Sydenham's Chorea. Investigations included an MRI head, which showed no abnormalities, and an Antistreptolysin $\mathrm{O}$ titre of +400 units $/ \mathrm{ml}$. This revealed a diagnosis of ARF, as per the revised Jones Criteria. She was started on prophylactic penicillin $\mathrm{V}$ and carbamazepine. Our patient made an improvement during her admission and was discharged with Cardiology and Neurology input.

In this case, we show that diseases that are typically associated with the developing world can also present in the UK. We demonstrate the importance of recognising the neuropsychiatric symptomatology of ARF. This will aid early diagnosis and management, which can significantly reduce the risk of long-term sequelae.

\section{G45(P) SEVERE NEUROLOGICAL IMPAIRMENT: TOWARDS AN INTERNATIONAL CONSENSUS-BASED DEFINITION}

\begin{abstract}
1,2,3 JA Allen, 1,2,3,4,5,6EJ Molloy, ${ }^{1,2,3} \mathrm{DM}$ McDonald. 'Department of Paediatrics, Children's Health Ireland at Tallaght, Dublin, Ireland; ${ }^{2}$ Discipline of Paediatrics, Trinity College Dublin, The University of Dublin, Dublin, Ireland; ${ }^{3}$ Trinity Research in Childhood Centre, Trinity College, The University of Dublin, Dublin, Ireland; ${ }^{4}$ Trinity Translational Medicine Institute, Trinity College, The University of Dublin, Dublin, Ireland; ${ }^{5}$ Neonatology, Coombe Women and Infants' University Hospital, Dublin, Ireland; ${ }^{6}$ Neonatology, Children's Health Ireland at Crumlin, Dublin, Ireland
\end{abstract}

10.1136/archdischild-2020-rcpch.32

Aims Following a thorough review of the literature, we have found significant inconsistency in the use of the term Severe 
Neurological Impairment (SNI) in the literature. We aimed to develop an international, consensus-based, multi-disciplinary definition of this term.

Methods The Delphi process was chosen to achieve consensus on the definition of SNI. We collaborated with experts in 5 countries (Ireland, the UK, the USA, Canada and Australia) to disseminate an invitation to other colleagues in neurodisability in their own region. We specified that a multi-disciplinary panel was required. Those who wished to participate were asked to email us, as facilitators of the process, to confirm their desire to take part. Participants were asked to further disseminate the invitation to other colleagues, thus employing a snow-balling effect in the recruitment of expert panellists.

The Delphi process proceeded over 3 rounds. Round 1 used free-text responses where panellists provided insight into their understanding of the term SNI. Responses were used to generate themes. In rounds 2 and 3 panellists were asked to rate their agreement with these themes in the definition of SNI. In the process of round 3 participants were provided with feedback on the previous round, including anonymous information on how the other panellists had voted as well as selected written feedback to provide an opportunity to consider other points of view. Items were brought forward to the final definition if they received more than $70 \%$ agreement, in line with accepted Delphi methodology. After round 3, a working definition of SNI was created. Further refinements were made based on comments from parent representatives and experts at an international conference.

Results Thirty-four multi-disciplinary panellists participated in round 1 of the process falling to 31 in round 3, a 9\% dropout rate. Fifteen themes were generated from responses in round 1 . Seven items were brought forward for inclusion in the final definition.

Conclusion We have created an international, multi-disciplinary, consensus-based definition of SNI. This definition can be used to improve consistency in reporting of research, ultimately leading to improved outcomes for this unique and vulnerable cohort of children.

\section{G46(P) PROGRESSION OF NEUROPHYSIOLOGICAL CHANGES IN A CHILD WITH SUBACUTE SCLEROSING PANENCEPHALITIS}

${ }^{1}$ A Lazenbury, ${ }^{1} \mathrm{~N}$ Bhangu, ${ }^{1} \mathrm{M}$ Illingworth, ${ }^{2} \mathrm{D}$ Allen, IJ Singh. ${ }^{1}$ Department of Paediatric Neurology, University Hospital Southampton, Southampton, UK; ${ }^{2}$ Department of Clinical Neurophysiology, University Hospital Southampton, Southampton, UK

10.1136/archdischild-2020-rcpch.33

Subacute sclerosing panencephalitis (SSPE) is classically associated with characteristic electroencephalogram (EEG) features of periodic and stereotyped high voltage discharges. We present the electroclinical evolution in a case of SSPE in whom the typical changes were not initially evident. The patient is a fully immunized seven-year old boy with a sixmonth history of increasingly frequent atonic drops with behavioural change and cognitive decline. His initial EEG showed continuous high amplitude 1-2 $\mathrm{Hz}$ sharp and slow wave activity, which were frontally dominant with little change on midazolam infusion. Eight days later, the EEG had a diffusely slow background with anterior sharp waves in a periodic/semi-periodic fashion, with complexes every 1-2 seconds.
A rapid neurocognitive decline ensued, characterized by fluctuating consciousness, visual impairment, aphasia and the emergence of dystonia, rendering him non-ambulant within weeks of hospital admission. The EEG evolved with a diffusely slow background with no periodic sharp waves, except occasionally in the temporal regions. MRI brain showed asymmetrical T2-weighted signal change in sub-cortical and deep white matter. Serial imaging demonstrated increasing widespread signal abnormality. SSPE was confirmed by CSF measles PCR. His EEG at ten weeks after presentation progressed to typical periodic sharp and slow wave complexes, occurring every 6-7 seconds.

The clinical course and EEG add weight to the growing body of evidence of SSPE occurring in a younger than expected cohort across the UK and Europe. SSPE is a neurodegenerative disorder where early illness is known to have a higher association with development of measles related sequelae. At a time when vaccination uptake is at an all time low, increased awareness of the burden of measles is crucial to facilitate improved health promotion and vaccination uptake worldwide. SSPE should be considered in any child presenting with regression and seizures even if the EEG and neuroimaging are not initially wholly supportive.

\section{G48(P) WRIST-WORN DEVICES TO IMPROVE ARM MOVEMENT IN HEMIPLEGIC CEREBRAL PALSY: PARTICIPATORY DESIGN WORKSHOPS}

${ }^{1} \mathrm{R}$ Brown, ${ }^{2} \mathrm{~J}$ Pearse, ${ }^{3} \mathrm{G}$ Edmonds, ${ }^{4} \mathrm{~T}$ Nappey, ${ }^{4} \mathrm{~K}$ Ladha, ${ }^{4} \mathrm{D}$ Jackson, ${ }^{4} \mathrm{Y}$ Guan, ${ }^{3,5} \mathrm{~A}$ Basu. ${ }^{1}$ Psychology, Newcastle University, Newcastle upon Tyne, UK; ${ }^{2}$ Occupational Therapy, Great North Childrens Hospital, Newcastle upon Tyne, UK; ${ }^{3}$ Institute of Health and Society, Newcastle University, Newcastle upon Tyne, UK; ${ }^{4}$ Computing Science, Newcastle University, Newcastle upon Tyne, UK; ${ }^{5}$ Paediatric Neurology, Great North Childrens Hospital, Newcastle upon Tyne, UK

\subsection{6/archdischild-2020-rcpch.34}

Background Hemiplegic Cerebral Palsy causes unilateral weakness and stiffness. Upper limb therapy at effective intensity is not accessible to most.

Aim Determine stakeholder views on design of an approach using wrist-worn devices and software (phone application) incorporating positive feedback and peer support, to encourage use of the affected arm and hand.

Method Five participatory design workshops, incorporating the views of 5 young people (YP) with hemiplegia and 13 typically developing (TD) (8-18 years) plus three parents, six researchers, three occupational therapists, one teacher and two paediatricians.

Results Four themes were noted: Ease/practical issues, Appearance/comfort, Incentives, and Access to participants. Ease/practical issues: Distinguishing between left and right devices proved challenging, so images of differing orientation were added. Concern about teachers' disapproval was resolved by producing an explanatory 'business card' for YP to hand out. Mobile phones are not required in school as the device and app can synchronise later, via Bluetooth. Concerns were addressed, regarding whether devices could gather secure information from arm movement, e.g. PIN numbers. Reassuringly, data is averaged over a minute, rendering this impossible. A parent asked whether GPs could use the data for other purposes (e.g. investigation of tremor) - but this would present challenges. Incentives: Games were a popular incentive, especially those with a competitive element. Inclusion of 